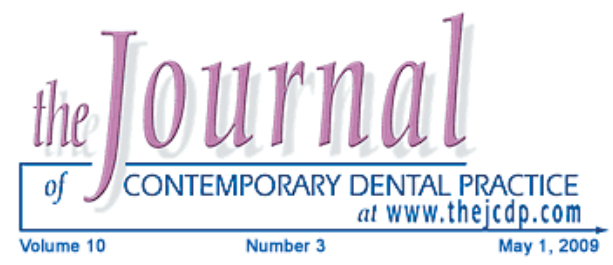

\title{
Effect of Posts on the Fracture Resistance of Load-cycled Endodontically-treated Premolars Restored with Direct Composite Resin
}

Hila Hajizadeh, DDS, MS;

Mohammad Sadegh Namazikhah, DMD, MSEd;

Mohammad Javad Moghaddas, DDS, MS;

Marjaneh Ghavamnasiri, DDS, MS; Sara Majidinia, DDS

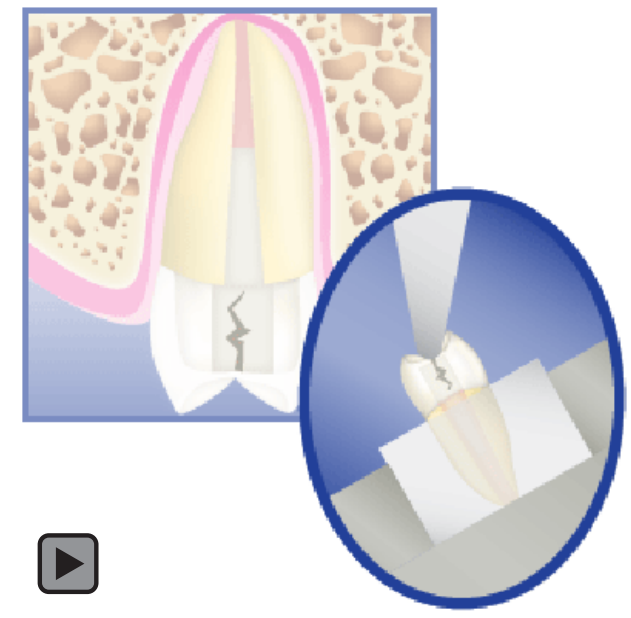

Abstract

Aim: The aim of this study was to investigate the fracture resistance and failure mode of premolars restored with composite resin using various prefabricated posts.

Methods and Materials: Sixty sound maxillary premolars were divided into four equal sized groups. All but the control group received endodontic treatment followed by placement of mesiodistocclusal (MOD) composite restorations (Tetric Ceram) as follows: Group T = no post, Group DT = fiber reinforced composite (FRC) post (DT Light), Group FL = prefabricated metal post (Filpost). The control group (C) had no cavities prepared. After thermal and load cycling, static load was applied at a $30^{\circ}$ angle until fracture. Failure modes were categorized as restorable and non-restorable. Data were analyzed using the analysis of variance (ANOVA) and Duncan tests $(\alpha=0.05)$.

Results: The mean values of fracture loads $(\mathrm{N})$ for all groups were: C (880 \pm 258$)$; T (691 \pm 239$)$; DT (865 \pm 269$)$; and FL $(388 \pm 167)$. Statistically significant differences $(P<0.05)$ were observed for all groups except between groups $\mathrm{C}$ and DT. The Chi Square test showed failure modes in groups $\mathrm{C}$ and DT were mostly restorable. The most non-restorable fractures were observed in group FT.

(C) Seer Publishing 
Conclusion: Intact teeth and the teeth restored with composite and quartz fiber posts had a similar fracture resistance and the failure modes were mostly restorable. The lowest fracture resistance and the most nonrestorable failures were observed in conjunction with metal posts.

Clinical Significance: The results of this in vitro study suggest the use of a quartz fiber post used in conjunction with an MOD composite resin restoration improves fracture resistance in an endodontically treated premolar.

Keywords: FRC post, prefabricated metal post, fracture resistance, composite resin restoration

Citation: Hajizadeh H, Namazikhah MS, Moghaddas MJ, Ghavamnasiri M, Majidinia S. Effect of Posts on the Fracture Resistance of Load-cycled Endodontically-treated Premolars Restored with Direct Composite Resin. J Contemp Dent Pract 2009 May; (10)3:010-017.

\section{Introduction}

Endodontically treated teeth with extensive loss of coronal structure can be problematic because of a significant reduction in their capacity to resist functional forces. The greatest incidence of vertical crown and root fractures occurs in endodontically treated teeth. The strength of these teeth is directly related to the amount of remaining sound tooth structure. ${ }^{1-4}$

The introduction of adhesive techniques has facilitated the preservation of maximum sound tooth structure. Although there have been previous studies that have examined the fracture strength of premolars restored with composite compared with that of intact premolars, the results have been conflicting. ${ }^{5-10}$

Post insertion may be necessary when a core build up does not provide sufficient retention for a final restoration. However, some studies dispute

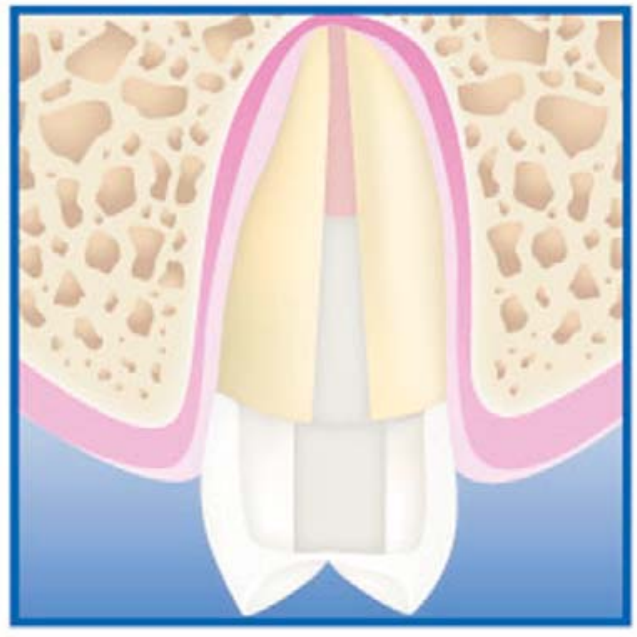

the reinforcement potential of posts. ${ }^{11,12}$ The use of metal-free posts with physical properties similar to dentin has become a focus of interest in dentistry. ${ }^{13,14}$

Some previous studies showed no significant difference in the fracture resistance of endodontically treated premolars with prefabricated metal posts, fiber reinforced composite (FRC) posts, and without posts after restoration with direct composite resin crowns. ${ }^{15,16}$ Nevertheless, a recent study revealed stainless steel posts lead to a higher stress concentration than glass fiber posts because of a significant difference between the elastic modulus of the steel and the surrounding structures. ${ }^{17}$ To avoid this problem a post and core should have the same elastic modulus as root dentin in order to distribute forces along the long axis of the post. ${ }^{18}$

Clinical trials have demonstrated extensive composite resin restorations used in conjunction with either FRC posts, or with prefabricated metal posts, were comparable to conventional post and core/crown combinations. ${ }^{19-22}$ The findings of one clinical trial indicated composite restoration with fiber posts in premolar teeth were more effective than amalgam in preventing root fracture but less effective in preventing secondary caries. ${ }^{23}$

Load cycling has been established as an essential research tool for testing adhesive restorations because the cyclic loading pattern is comparable to actual physiological function..$^{24}$ In an "artificial mouth" $1,200,000$ chewing cycles can simulate five years of clinical service of a restoration. ${ }^{14}$ 
However, some controversy exists in the literature regarding the application of the load angle for premolar teeth. ${ }^{7,25,26}$

The purposes of this study were to evaluate the influence of different posts on fracture resistance and the failure mode of endodontically treated premolars restored with mesiodistocclusal (MOD) direct composite resin restorations.

\section{Methods and Materials}

Sixty extracted, single-rooted human maxillary premolars without caries, wear, or fractures were used for this study. Selection criteria included similar size, absence of caries, and fracture lines in either the crown or the root. The teeth were examined using a stereomicroscope under 10X magnification to confirm they were free of cracks. The selected teeth were then randomly divided into four groups $(n=15)$ and stored in water until processing.

No cavities were prepared in the first group which served as control (Group C), while all other groups were endodontically treated. Radiographic images were taken and an access opening was prepared to facilitate instrumentation of the root canals to an ISO 35 (2\% taper in all teeth for standardization) using the Flex Master-Kit (VDW, Munich, Germany). After irrigation with sodium hypochlorite, the canals were obturated with laterally condensed gutta percha (Maillefer, Ballaigues, Switzerland) and AH26 resin sealer (DeTrey, Zurich, Switzerland).
Next, the root of each tooth was embedded in an aluminum cylinder (external diameter $25 \mathrm{~mm}$, height $20 \mathrm{~mm}$ ) up to $2 \mathrm{~mm}$ below the cementoenamel junction (CEJ) using Unifest II autopolymerizing acrylic resin (GC, Tokyo, Japan). MOD cavities were then prepared in each tooth. Diamond rotary cutting instruments in a high-speed handpiece under copious air-water cooling were used for cavity preparations. Burs were replaced after every five preparations. The buccolingual width on the occlusal surface was two thirds of the intercuspal distance $(3.0 \mathrm{~mm})$ and $4.0 \mathrm{~mm}$ deep on the gingival floor which was set $1.0 \mathrm{~mm}$ above the CEJ. Each group was treated differently as described below.

Group $\mathbf{C}=$ No cavity preparation or restoration (control) was done.

Group $\mathbf{T}=$ No post was placed. Excite bonding agent (Ivoclar/Vivadent, Schaan, Liechtenstein) was applied according to the manufacturer's instructions. Next, a metal Tofflemire matrix band (KerrHawe, Bioggio, Switzerland) was positioned on the tooth and Tetric Ceram hybrid composite resin (Ivoclar/ Vivadent) was incrementally placed in the cavity and each increment light polymerized for 40 seconds using an Optilux 500 curing unit (Demetron/Kerr, Orange, CA, USA). The restorations were then contoured anatomically and polished.

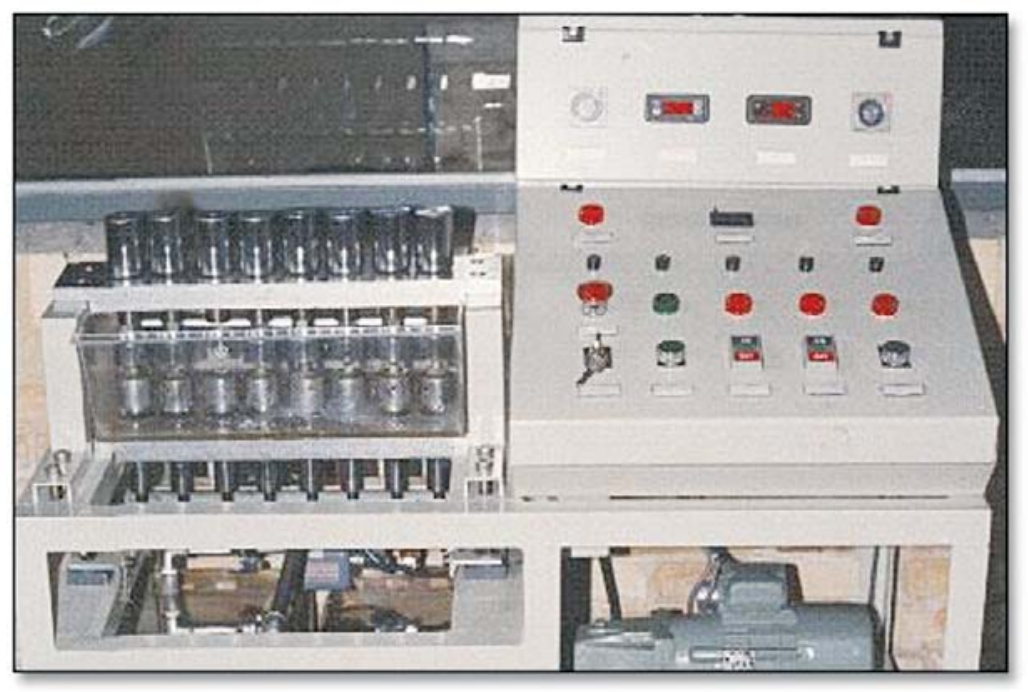

Figure 1. The load cycling machine. 
Group DT = DT Light prefabricated, $1.8 \mathrm{~mm}$ diameter quartz fiber posts (RTD, St. Egreve, France) were cleaned with ethanol, dried, and silanized for 30 seconds with Scotchbond Ceramic Primer (3M ESPE, St. Paul, MN, USA). Root canals were then prepared $8 \mathrm{~mm}$ below the orifice using a twist drill (RTD). The canals were preconditioned with ED Primer self-etching primer (Kuraray Medical Inc, Okayama, Japan) for 60 seconds. Any remaining primer in the apical segment of the canal was removed with an absorbent paper cone. Panavia F 2.0 resin cement (Kuraray Medical Inc.) was mixed for 20 seconds and applied to the post surface before seating using finger pressure. The cement was light polymerized for 40 seconds from the occlusal direction. The composite restoration was then placed in the same manner as was done for Group T.

Group $\mathrm{FL}=$ This group was treated like the Group DT except that a Filpost (Filhol Dental, Gloucestershire, UK) prefabricated metal post was used instead of a quartz fiber post.

All groups were exposed to a cyclic loading for 720,000 cycles. The load cycling device was designed and fabricated at Mashhad University of Medical Sciences, Mashhad, Iran (Figure 1).

Each specimen was load cycled on the occlusal surface with the load direction nearly parallel to the long axis of the tooth at a frequency of $3 \mathrm{~Hz}^{24}$ using a $10 \mathrm{~mm}$ diameter steatite ceramic ball ${ }^{27}$ (Hoechst Ceramtec, Wunsiedel, Germany) with a $16 \mathrm{lb}$ load value ${ }^{26}$ (Figure 2). During load cycling all specimens were subjected to continuous thermal cycling between $5^{\circ} \mathrm{C}$ and $55^{\circ} \mathrm{C}$ for 60 seconds each. ${ }^{28}$

The specimens were then submitted to the fracture resistance test using a universal testing machine (Instron, Canton, MA, USA) with a $2 \mathrm{~mm}$ diameter steel sphere crosshead welded to a tapered shaft and applied to the specimens at a constant speed of $5 \mathrm{~mm} / \mathrm{min}$ and at an angle of 30 degrees to the long axis of the tooth. ${ }^{15}$ The site of loading was the central fissure of the occlusal surface in the direction of the buccal cusp (Figure 3).

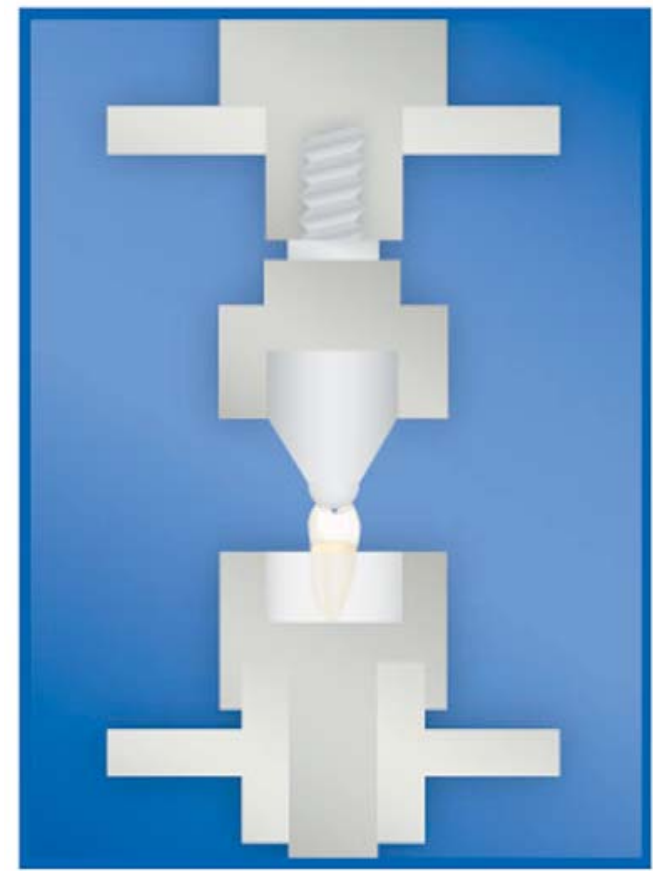

Figure 2. The schematic illustration of specimens in the load cycling machine.

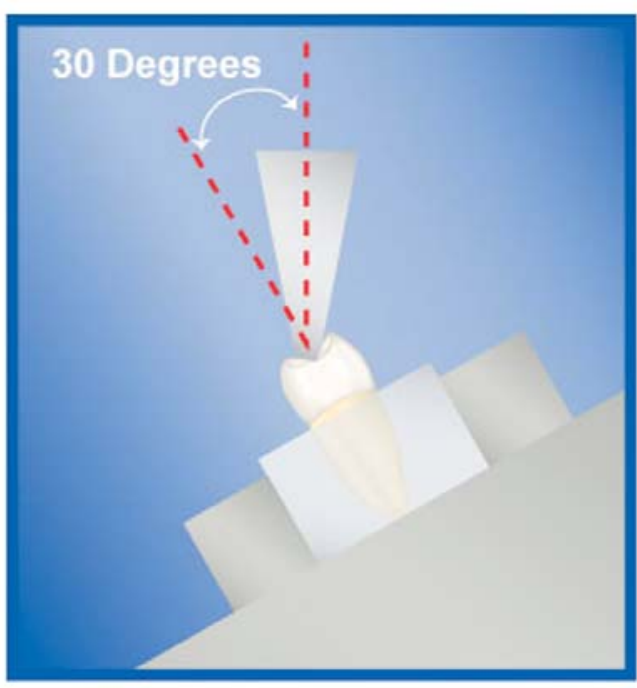

Figure 3. The schematic illustration of applying load during fracture test.

Fractured specimens were assessed for failure modes: "Restorable failures" including adhesive failures above the CEJ and "Non-restorable failures" including vertical root fractures below the CEJ. ${ }^{29}$ 
The data were statistically analyzed by oneway analysis of variance (ANOVA) with SPSS 13 (SPSS Inc., Chicago, IL, USA). Effects with a $P$ value not exceeding 0.05 were considered significant. Whenever a significant difference was observed, effects were further explored by Duncan Multiple Range test. Chi-Square test was also used to compare the frequencies of failure mode of specimens $(\alpha=0.05)$.

\section{Results}

Specimens fractured at failure loads of $388-880$ $\mathrm{N}$ (Table 1). The ANOVA showed a significant difference among the groups $(\mathrm{P}<0.05)$.

The Duncan Multiple Range test showed no significant difference between Groups C and DT $(P>0.05)$, while there were significant differences

Table 1. Failure load data in the four groups.

\begin{tabular}{|l|c|c|c|}
\hline \multicolumn{1}{|c|}{ Group } & N & Mean failure load (N) & SD \\
\hline C & 15 & $880.15 \alpha$ & 258.09 \\
\hline T & 15 & $691.4 \beta$ & 239.00 \\
\hline DT & 15 & $865.66 \alpha$ & 269.96 \\
\hline FL & 15 & $388.29 \gamma$ & 167.27 \\
\hline Similar letters show no significant difference $(P>0.05)$.
\end{tabular}

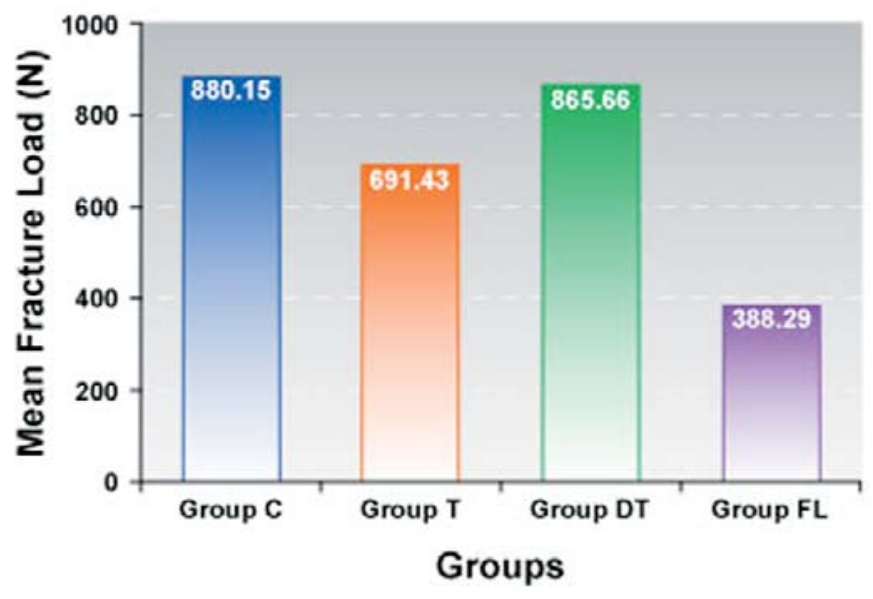

Figure 4. Mean fracture load to failure in all groups.

Table 2. Classification of specimens from each qroup based on fracture mode.*

\begin{tabular}{|l|c|c|c|c|}
\hline \multirow{2}{*}{\multicolumn{2}{c|}{ Fracture Mode }} & \multicolumn{4}{|c|}{ Group } \\
\cline { 2 - 5 } & C & T & DT & FL \\
\hline Restorable & $9(60 \%)$ & $9(60 \%)$ & $13(86 \%)$ & $3(20 \%)$ \\
\hline Non-Restorable & $6(40 \%)$ & $6(40 \%)$ & $2(14 \%)$ & $12(80 \%)$ \\
\hline -Unit = teeth & & & \\
\hline
\end{tabular}


between Groups $\mathrm{C}$ and T, C and FL, T and DT, T and $F L$, and DT and FL $(P<0.05)$.

The highest fracture resistance was related to Groups C and DT while the lowest one represented Group FL. Figure 4 shows the mean fracture load for all groups.

Restorable fracture in Groups C, T, DT, and FL were $60 \%, 60 \%, 86 \%$, and $20 \%$, respectively (Table 2). The Chi-Square test showed a significant difference in result frequencies of restorable/non-restorable failure modes among groups $(\mathrm{P}<0.05)$.

\section{Discussion}

The present study used endodontically treated maxillary premolars to determine the appropriateness of different restoration methods since these teeth present an unfavorable anatomic shape, crown value, and crown/ root proportion, making them more susceptible to cusp fractures than other posterior teeth when submitted to occlusal load application. ${ }^{30}$ Additionally, endodontic access associated with removal of pulp chamber walls and root dentin appears to be directly responsible for the greater fragility of endodontically treated teeth. ${ }^{31}$

A $16 \mathrm{lb}$ load value was chosen for the present study which was within the physiologic chewing force range. ${ }^{26}$ There is controversy regarding the application of the load angle for premolar teeth in a load cycling device. ${ }^{7,25,26}$ Specimens received the applied load parallel to their long axes in the load cycling device. ${ }^{24}$

Similar to the result of a previous study, ${ }^{10}$ in the present study, only $60 \%$ of the fractures in intact teeth (Group C) were restorable. Oliveria et al. ${ }^{2}$ claimed it is unlikely loading stress could be concentrated on any particular area of an intact tooth.

The present finding showed no significant difference in fracture resistance of intact teeth (Group C) and fiber post composite resin restored teeth (Group DT). The few number of root fractures in teeth restored with fiber post and composite concurs with retrospective and prospective studies. ${ }^{21,22}$ Salameh et al. ${ }^{32}$ also suggested the post could contribute to the

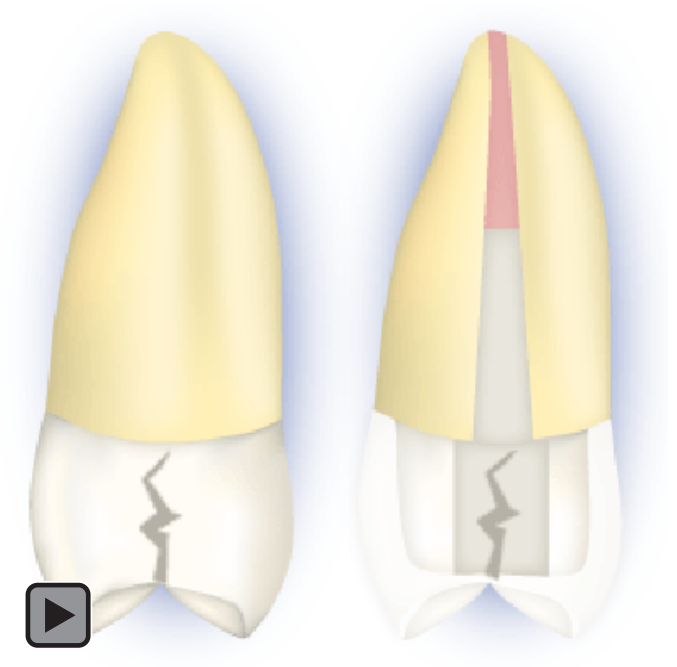

reinforcement and strengthening of pulpless maxillary premolars and placement of posts improved the fracture mode from non-restorable to restorable patterns. Sorrentino et al. ${ }^{3,33}$ revealed endodontically treated maxillary premolars restored with fiber posts exhibited predominantly restorable fractures.

Clinically the less rigid composite restoration will probably wear more than the amalgam and full crown restorations; therefore, composite resin might transfer less stress to the fiber posts and the remaining tooth structure. In addition, a fiber post has elastic modulus similar to tooth structure. ${ }^{23}$ This could account for the absence of root fractures in teeth restored with fiber posts and composite resin.

In general, the findings of this study showed the fracture load value in Group T (no post) was significantly lower than intact teeth. Other studies also have detected higher fracture strength for intact teeth when compared to those with adhesively bonded MOD restorations. ${ }^{6,34-36}$

The mean fracture load value of Group T (no post) was significantly lower than Group DT (FRC post). This probably can be attributed to the bonding mechanism starting from the buccal wall and progressing toward the lingual wall within an adhesive MOD restoration to "splint" the tooth structure. The presence of an FRC post decreases the splinting distance which may result in a higher fracture strength of the tooth. 
The results of the present study also showed the lowest fracture resistance in Group FL (metal post). Differences in the elastic modulus of the post and the composite resin may be responsible for this finding. ${ }^{17}$ The fracture value in Groups $\mathrm{T}$ (no post) and FL (metal post) also showed a significant difference. The thicker bulk of a composite resin restoration with no post might result in the higher fracture resistance than with the use of a metal post. ${ }^{15}$

A prefabricated quartz fiber post with an MOD composite resin restoration appears to be a reasonable choice to successfully restore endodontically treated maxillary-premolars because the failure mode was rarely nonrestorable using this restorative strategy.

Traditional thinking that a post is only placed to retain a core and serves no other purpose may no longer be valid. This may have been true with metal posts, but there is growing evidence fiber posts provide the addition benefit of increased fracture resistance. Fiber posts have opened the door to additional indications. Since they do not appear to be associated with root fractures like metal posts, they should probably be placed in most situations where fracture in the cervical area is a concern. As long as no additional dentin is removed, there appears to be no downside and they may help to prevent clinical failure. The same is true for post placement after completion of endodontic treatment through an existing crown.

Only two post systems and one composite material were tested in the present study. Caution is advised when attempting to extrapolate such a limited sample into a principle that can be applied globally to clinical situations. The fracture of restorations may be influenced by other factors, and despite the importance of short laboratory studies only long-term clinical trials can provide the answers to some remaining questions.

\section{Conclusion}

Intact teeth and the teeth restored with composite and quartz fiber posts had a similar fracture resistance. Failure modes in the two mentioned groups were mostly restorable. The lowest fracture resistance and the most non-restorable failures were observed in composite resin restorations in conjunction with metal posts.

\section{Clinical Significance}

The results of this in vitro study suggest a quartz fiber post used in conjunction with an MOD composite resin restoration improves fracture resistance in an endodontically treated premolar. 


\section{References}

1. Mondelli J, Sene F, Ramos RP, Benetti AR. Tooth structure and fracture strength of cavities. Braz Dent J 2007; 18(2):134-8.

2. Oliveira FC, Denehy GE, Boyer DB. Fracture resistance of endodontically prepared teeth using various restorative materials. J Am Dent Assoc 1987; 115(1):57-60.

3. Sorrentino R, Monticelli F, Goracci C, Zarone F, Tay FR, Garcia-Godoy F, Ferrari M. Effect of postretained composite restorations and amount of coronal residual structure on the fracture resistance of endodontically-treated teeth. Am J Dent 2007; 20(4):269-74.

4. Trabert KC, Caput AA, bou-Rass M. Tooth fracture-a comparison of endodontic and restorative treatments. J Endod 1978; 4(11):341-5.

5. Cotert HS, Sen BH, Balkan M. In vitro comparison of cuspal fracture resistances of posterior teeth restored with various adhesive restorations. Int J Prosthodont 2001; 14(4):374-8.

6. Habekost LV, Camacho GB, Azevedo EC, Demarco FF. Fracture resistance of thermal cycled and endodontically treated premolars with adhesive restorations. J Prosthet Dent 2007; 98(3):186-92.

7. Shor A, Nicholls JI, Phillips KM, Libman WJ. Fatigue load of teeth restored with bonded direct composite and indirect ceramic inlays in MOD class II cavity preparations. Int J Prosthodont 2003; 16(1):64-9.

8. Soares PV, Santos-Filho PC, Queiroz EC, Araujo TC, Campos RE, Araujo CA, Soares CJ. Fracture resistance and stress distribution in endodontically treated maxillary premolars restored with composite resin. J Prosthodont 2008; 17(2):114-9.

9. Soares PV, Santos-Filho PC, Martins LR, Soares CJ. Influence of restorative technique on the biomechanical behavior of endodontically treated maxillary premolars. Part I: Fracture resistance and fracture mode. J Prosthet Dent 2008; 99(1):30-7.

10. Yamada $Y$, Tsubota $Y$, Fukushima S. Effect of restoration method on fracture resistance of endodontically treated maxillary premolars. Int J Prosthodont 2004; 17(1):94-8.

11. Caputo AA, Standlee JP. Pins and posts--why, when and how. Dent Clin North Am 1976; 20(2): 299-311.

12. Sornkul E, Stannard JG. Strength of roots before and after endodontic treatment and restoration. J Endod 1992; 18(9):440-3.

13. Akkayan B, Gulmez T. Resistance to fracture of endodontically treated teeth restored with different post systems. J Prosthet Dent 2002; 87(4):431-7.

14. Heydecke G, Butz F, Hussein A, Strub JR. Fracture strength after dynamic loading of endodontically treated teeth restored with different post-and-core systems. J Prosthet Dent 2002; 87(4):438-45.

15. Fokkinga WA, Le Bell AM, Kreulen CM, Lassila LV, Vallittu PK, Creugers NH. Ex vivo fracture resistance of direct resin composite complete crowns with and without posts on maxillary premolars. Int Endod J 2005; 38(4):230-7.

16. Krejci I, Duc O, Dietschi D, de CE. Marginal adaptation, retention and fracture resistance of adhesive composite restorations on devital teeth with and without posts. Oper Dent 2003; 28(2): 127-35.

17. Barjau-Escribano A, Sancho-Bru JL, Forner-Navarro L, Rodriguez-Cervantes PJ, Perez-Gonzalez A, Sanchez-Marin FT. Influence of prefabricated post material on restored teeth: fracture strength and stress distribution. Oper Dent 2006; 31(1):47-54.

18. Dietschi D, Romelli M, Goretti A. Adaptation of adhesive posts and cores to dentin after fatigue testing. Int J Prosthodont 1997; 10(6):498-507.

19. Creugers NH, Mentink AG, Fokkinga WA, Kreulen CM. 5-year follow-up of a prospective clinical study on various types of core restorations. Int J Prosthodont 2005; 18(1):34-9.

20. Creugers NH, Kreulen CM, Fokkinga WA, Mentink AG. A 5-year prospective clinical study on core restorations without covering crowns. Int J Prosthodont 2005; 18(1):40-1.

21. Ferrari M, Vichi A, Mannocci F, Mason PN. Retrospective study of the clinical performance of fiber posts. Am J Dent 2000; 13(Spec No):9B-13B.

22. Mannocci F, Bertelli E, Sherriff M, Watson TF, Ford TR. Three-year clinical comparison of survival of endodontically treated teeth restored with either full cast coverage or with direct composite restoration. J Prosthet Dent 2002; 88(3):297-301. 
23. Mannocci F, Qualtrough AJ, Worthington HV, Watson TF, Pitt Ford TR. Randomized clinical comparison of endodontically treated teeth restored with amalgam or with fiber posts and resin composite: five-year results. Oper Dent 2005; 30(1):9-15.

24. DeLong R, Douglas WH. Development of an artificial oral environment for the testing of dental restoratives: bi-axial force and movement control. J Dent Res 1983; 62(1):32-6.

25. Bolhuis $P$, deGee A, Feilzer A. Influence of fatigue loading on four post-and-core systems in maxillary premolars. Quintessence Int 2004; 35(8):657-67.

26. Fissore B, Nicholls JI, Yuodelis RA. Load fatigue of teeth restored by a dentin bonding agent and a posterior composite resin. J Prosthet Dent 1991; 65(1):80-5.

27. Kern M, Strub JR, Lu XY. Wear of composite resin veneering materials in a dual-axis chewing simulator. J Oral Rehabil 1999; 26(5):372-8.

28. Koutayas SO, Kern M, Ferraresso F, Strub JR. Influence of design and mode of loading on the fracture strength of all-ceramic resin-bonded fixed partial dentures: an in vitro study in a dual-axis chewing simulator. J Prosthet Dent 2000; 83(5):540-7.

29. Fennis WM, Tezvergil A, Kuijs RH, Lassila LV, Kreulen CM, Creugers NH, Vallittu PK. In vitro fracture resistance of fiber reinforced cusp-replacing composite restorations. Dent Mater 2005; 21(6):565-72.

30. Fennis WM, Kuijs RH, Kreulen CM, Roeters FJ, Creugers NH, Burgersdijk RC. A survey of cusp fractures in a population of general dental practices. Int J Prosthodont 2002; 15(6):559-63.

31. Ross IF. Fracture susceptibility of endodontically treated teeth. J Endod 1980; 6(5):560-5.

32. Salameh Z, Sorrentino R, Ounsi HF, Goracci C, Tashkandi E, Tay FR, Ferrari M. Effect of different all-ceramic crown system on fracture resistance and failure pattern of endodontically treated maxillary premolars restored with and without glass fiber posts. J Endod 2007; 33(7):848-51.

33. Sorrentino R, Salameh Z, Zarone F, Tay FR, Ferrari M. Effect of post-retained composite restoration of MOD preparations on the fracture resistance of endodontically treated teeth. J Adhes Dent 2007; 9(1):49-56.

34. Santos MJ, Bezerra RB. Fracture resistance of maxillary premolars restored with direct and indirect adhesive techniques. J Can Dent Assoc 2005; 71(8):585.

35. St-Georges AJ, Sturdevant JR, Swift EJ, Jr., Thompson JY. Fracture resistance of prepared teeth restored with bonded inlay restorations. J Prosthet Dent 2003; 89(6):551-7.

36. Habekost LV, Camacho GB, Pinto MB, Demarco FF. Fracture resistance of premolars restored with partial ceramic restorations and submitted to two different loading stresses. Oper Dent 2006; 31(2):204-11.

\section{About the Authors}

Hila Haiizadeh. DDS, MS

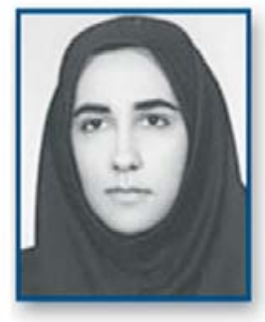

Dr. Hajizadeh is an Assistant Professor in the Department of Restorative Dentistry of the Mashhad Dental School and Dental Research Center at Mashhad University of Medical Sciences in Mashhad, Iran.

e-mail: hajizadehh@mums.ac.ir 
Mohammad Sadegh Namazikhah, DMD, MSEd

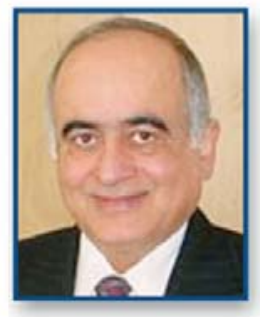

Dr. Namazikhah is a Professor and former Chair of the Department of Endodontics and now serves as the Director of the Advanced Endodontic Program at the University of Southern California School of Dentistry in Los Angeles, CA, USA.

e-mail: namazikhah.sadegh@verizon.net

Mohammad Javad Moghaddas, DDS, MS

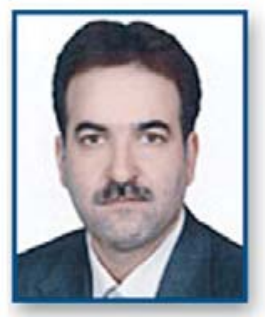

Dr. Moghaddas is an Assistant Professor in the Department of Restorative Dentistry of the Mashhad Dental School and Dental Research Center at Mashhad University of Medical Sciences in Mashhad, Iran.

e-mail: moghaddasmj@mums.ac.ir

\section{Marjaneh Ghavamnasiri, DDS, MS}

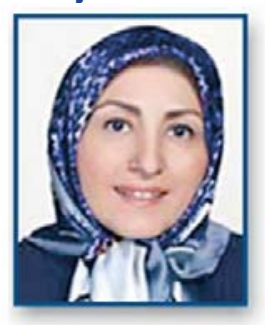

Dr. Ghavamnasiri is a Professor and the Director of the Postgraduate Program in the Department of Restorative Dentistry of the Mashhad Dental School and Dental Research Center at Mashhad University of Medical Sciences in Mashhad, Iran.

e-mail: ghavamnasirim@mums.ac.ir

\section{Sara Majidinia, DDS}

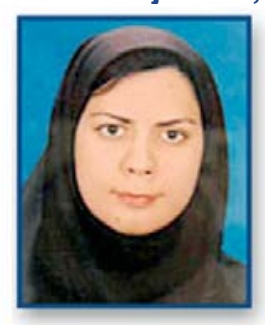

Dr. Majidinia is a general dentist in private practice in Mashhad, Iran.

e-mail: sara_majidinia@yahoo.com 